\title{
Changes in cholinergic sweat gland activation in diabetic neuropathy identified by computerised sweatspot analysis
}

\author{
D.M. Levy, D. A. Rowley and R. R. Abraham \\ Diabetic Neuropathy Research Group, Department of Diabetes and Endocrinology, Central Middlesex Hospital, London, UK
}

\begin{abstract}
Summary. Peripheral small-fibre denervation has been reported to result in decreased activation of eccrine sweat glands to muscarinic cholinergic agents. Using computerised image-analysis of pilocarpine-activated sweatspot prints of a $4 \mathrm{~cm}^{2}$ area of the dorsum of the foot in 79 randomly selected diabetic patients we have identified a group of neuropathic patients $(18 \%)$ with decreased sweatspot activation $\left(<20 / \mathrm{cm}^{2}\right)$, and a smaller group $(6 \%)$ of younger patients with less marked neuropathy who had increased activation $\left(>132 / \mathrm{cm}^{2}\right)$, probably resulting from denervation supersensitivity. The associations between sweatspot density and other conventional tests of peripheral nerve function were weak. The prevalence of abnormal sweatspot density, $24 \%$,
\end{abstract}

was similar to that of other tests, except thermal thresholds at the feet $(35-37 \%)$, which were not correlated with sweatspot activation, suggesting that diabetic neuropathy has differing effects on afferent and efferent small fibres. The method is rapid and reproducible (median coefficient of variation $14 \%$ ) and its ability to identify patients with increased, as well as decreased, peripheral nerve function may be of value in the characterisation and longitudinal follow-up of smallfibre abnormalities in diabetes.

Key words: Autonomic neuropathy, Sweat glands, Sweating, Denervation supersensitivity, Electrophysiology, Sensory thresholds.
Sweat secretion in response to local cholinergic stimulation is known to depend on an intact postganglionic sympathetic supply to the sweat glands $[1,2]$. Early qualitative studies confirmed that human sweat glands were unresponsive to cholinergic agents immediately after acute denervation $[3,4]$. The structure of the glands is preserved [5] and acetylcholinesterase disappears [6], suggesting that cholinergic unresponsiveness after complete denervation is neural in aetiology. In chronic diabetic neuropathy manual counting $[7,8]$ confirmed a decrease in the number of activatable sweat glands in the feet which paralleled the deficit in other small nerve fibres [9]. Kennedy and Navarro [10] recently used imageanalysis to count sweat spot imprints over a distal $1 \mathrm{~cm}^{2}$ area of the foot, and found that this method was more sensitive in the detection of subnormal sweatspot activation than manual counting. Many of the patients in their study were enrolled in a pancreatic transplantation programme, and had evidence of advanced peripheral and autonomic neuropathy.

Low et al. [11], using nicotinic cholinergic stimulation of sweat glands, found that while most neuropathic patients had reduced or absent sweat responses to acetylcholine, there was a small proportion with prolonged, supranormal responses, suggesting the presence of denervation supersensitivity. This phenomenon had not, until then, been observed in either acute denervation in experimental diabetes or in chronic human diabetic neuropathy.

In this study we have chosen to study randomly selected diabetic patients from a general diabetic clinic. The aims were to increase the precision of sweatspot counting by using a computerised method and by measuring pilocarpine-activated sweatspots over a larger area $\left(4 \mathrm{~cm}^{2}\right)$ of the foot, in order to characterise abnormal sweat gland activation, and to relate the measurements to other neurological tests in this population.

\section{Subjects and methods}

\section{Subjects}

Seventy-nine diabetic patients were selected at random from our clinic database of approximately 1,400 patients; 53 normal, agematched subjects were also studied. Their clinical characteristics are given in Table 1 . Thirty diabetic patients were considered to have symptomatic peripheral neuropathy, defined as any distal symmetri- 
Table 1. Clinical characteristics of the normal and diabetic subjects

\begin{tabular}{lll}
\hline & $\begin{array}{l}\text { Normal } \\
\text { subjects }\end{array}$ & $\begin{array}{l}\text { Diabetic } \\
\text { subjects }\end{array}$ \\
\hline Number & 53 & 78 \\
Age (years) & $45.6(12.0,19-79)$ & $43.6(14.9,17-66)$ \\
Gender (Male:Female) & $26: 27$ & 52.26 \\
Type 1:Type 2 & - & $43: 35$ \\
Diabetes duration (years) & - & $11.8(9.2,1-34)$ \\
$\begin{array}{l}\text { Symptomatic peripheral } \\
\text { neuropathy (\%) }\end{array}$ & - & $30(38 \%)$ \\
$\quad$ Age (years) & & $52.1(11.7,23-66)$ \\
$\quad \begin{array}{l}\text { Diabetes duration (years) } \\
\text { Synptomatic autonomic } \\
\text { neuropathy (\%) }\end{array}$ & - & $12.5(9.6,1-30)$ \\
$\quad$ Age (years) & & $25(32 \%)$ \\
$\begin{array}{l}\text { Diabetes duration (years) } \\
\text { Glycated haemoglobin (\%) }\end{array}$ & - & $50.4(12.7,21-66)$ \\
(<8.0\%) & $13.2(10.5,2-34)$ \\
$\begin{array}{l}\text { Random blood glucose } \\
\text { (mmol/l) }\end{array}$ & $11.2(2.8,6.5-16.5)[62]$ \\
\hline
\end{tabular}

Data are given as mean (SD, range) [], No. of subjects

cal numbness, paraesthesiae or pain in the lower limb, and 25 to have symptomatic autonomic neuropathy (sweating abnormalities, postural hypotension, gastrointestinal symptoms, impotence). Subjects were asked to stop using any skin preparation on the feet for 2 weeks before the test. Tests were performed in a warm room, $21-24^{\circ} \mathrm{C}$, with the subject semi-recumbent. Foot temperature was maintained at $30-34^{\circ} \mathrm{C}$, using convective heating if necessary. Capillary blood glucose measurements were made before the test (Glucometer II/Glucostix, Ames, Stoke Poges, UK) and venous blood taken for glycated haemoglobin (affinity chromatographic method, reference range $<8.0 \%$ ) at the end of testing.

\section{Indicator method}

The indicator bromophenol blue changes colour in aqueous alkaline media from light tan to dark blue [12]. Filter papers (Whatman Type 1, diameter $4.25 \mathrm{~cm}$ Maidstone, UK) were soaked in a $4 \%$ (weight/volume) solution of bromophenol blue indicator (BDH Poole, UK Catalogue No.20015, pH reference range 2.8-4.6) in acetone, rapidly air dried, and cut into semicircles.

\section{Sweat induction}

Sweating was stimulated over the right extensor digitorum brevis muscle with a battery-powered, rechargable constant-current generator (Wescor sweat inducer 3600, Chemlab Scientific Instruments, Romford, UK) developing $1.5 \mathrm{~mA}$ for $5 \mathrm{~min}$, in general paediatric use as a sweat-inducer for cystic fibrosis testing. $1 \%$ pilocarpine nitrate (Sigma Poole, UK Catalogue No.P6503) was incorporated into $2.5 \mathrm{~cm}$ diameter disks made of $4 \%$ agar gel. These were placed in the recessed iontophoresis electrodes; blank agar disks were used for the indifferent electrode (cathode) which was placed above the right ankle.

After iontophoresis, the electrodes were removed and timing started. In preliminary tests, very few sweatspots were found to be activated immediately after iontophoresis, and the first imprint was therefore taken at $4 \mathrm{~min}$, and then at 4 -min intervals up to $20 \mathrm{~min}$. In order to record sweatspots from the same anatomical area, the middle of the straight edge of the semicircular indicator paper was aligned with a mark made around the anode. The paper was applied to the skin for $60 \mathrm{~s}$ with a $2.5 \mathrm{~cm}^{2}$ block of foam rubber. The procedure was approved by the Ethics Committce of Parkside Health Authority.

\section{Image storage and analysis}

The indicator papers were photographed at three times enlargement with a Polaroid camera, using Polaroid type 669 colour film, and analysed by computerised image analysis (Quantimet 970, Cambridge, UK). Scaling and contrast sensitivity were established before each measuring session, and the image initially scanned four times in order to minimise background artefact, before presentation to the operator on a video display. Each sweatspot was outlined and its maximum diameter measured. The area of interest was outlined by optical mouse, excluding photographic artefacts. The derived measurements were produced in hard copy; those used in the current study were sweatspot density $\left(\mathrm{no} / \mathrm{cm}^{2}\right)$, and mean maximum diameter of sweatspots $(\mathrm{mm})$.

\section{Neurological tests}

The following additional tests were performed ( $\%$ of patients): common peroneal motor conduction velocity [13] (78\%); vibration perception thresholds (Biothesiometer) [14] (81\%); Marstock thermal thresholds [15] $(94 \%)$; autonomic function tests [16] (respiratory RR variation ( $96 \%$ ); Valsalva ratio $(81 \%)$; $30: 15$ ratio ( $96 \%$ ); lying and standing blood pressure (random zero sphygmomanometer) $(88 \%))$.

\section{Statistical analysis}

All data were normally distributed. Comparisons between groups were made using Student's $t$-test and correlations using Pearson correlation coefficients. Linear least squares regression analysis was used for evaluation of individual associations, and the Mann-Whitney U-test for sub-group analysis.

\section{Results}

\section{Normal subjects}

Mean sweatspot density increased from $65 / \mathrm{cm}^{2}$ at $4 \mathrm{~min}$ to $76 / \mathrm{cm}^{2}(\mathrm{SD} 28)$ at 12,16 and $20 \min (p<0.001)$. Since there was no change after $12 \mathrm{~min}$, the mean of the last three measurements was used. This yields an upper $95 \%$ confidence limit for the normal range of $132 / \mathrm{cm}^{2}$, and a lower limit of $20 / \mathrm{cm}^{2}$. Mean sweatspot diameter remained constant at $0.303 \mathrm{~mm}$ (SD 0.055) from 8-20 min. Mean sweatspot density and size were significantly correlated $\left(r^{2}=0.38\right.$, $p<0.001$ ) (Fig. 1 a). Measurements were lower in females than males (density: male $83 / \mathrm{cm}^{2}$, female $69 / \mathrm{cm}^{2}, p=0.09$; size: male $0.330 \mathrm{~mm}$, female $0.272 \mathrm{~mm}, p<0.001$ ). Neither mean sweatspot density nor size changed with age in normal subjects. Mean sweatspot diameter, but not density, was strongly related to both height and weight (height: $r^{2}=0.26, p=0.002$; weight: $r^{2}=0.20, p=0.008$ ).

\section{Diabetic patients}

Two subjects with severe symptomatic neuropathy had no detectable sweatspots. There was no difference in sweatspot activation between 8 and $20 \mathrm{~min}\left(74 / \mathrm{cm}^{2}\right)$. Sweatspot density and size was similar in normal and diabetic subjects. As in normal subjects, sweatspot density and size 

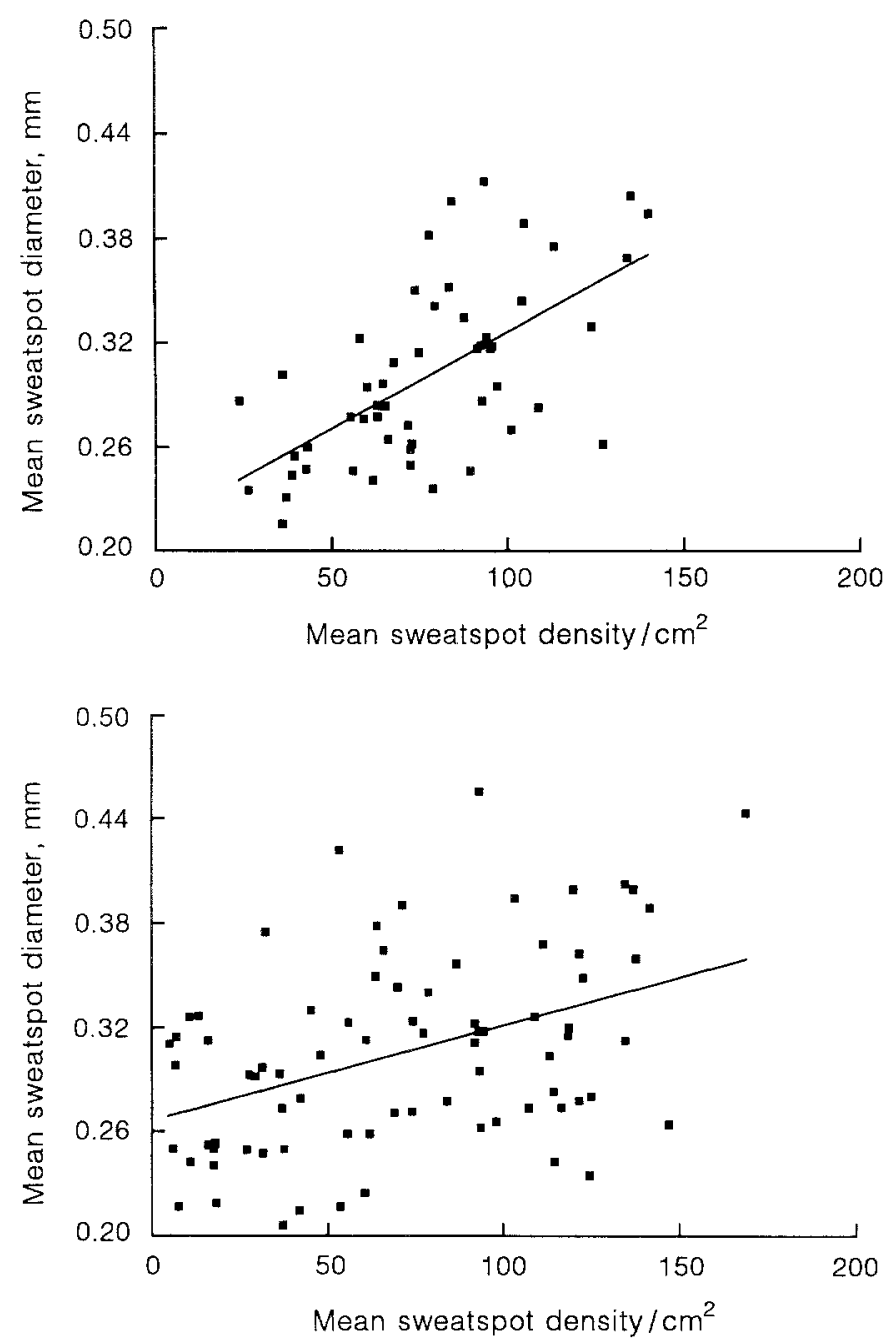

Fig. 1. The relationship between pilocarpine-activated sweatspot density and maximum sweatspot diameter in normal subjects (upper plot: $r^{2}=0.38, p<0.001$ ) and diabetic subjects (lower plot: $r^{2}=0.16$, $p<0.001$ )

were also significantly correlated $\left(r^{2}=0.16, p<0.001\right)$ (Fig. 1 b). Density, but not size, decreased with age (density: $r^{2}=0.12, p=0.002$; size: $r^{2}=0.03, p=0.12$ ); neither showed any association with diabetes duration. As in normal subjects, mean sweatspot diameter was related both to height $\left(r^{2}=0.16, p=0.001\right)$ and to weight $\left(r^{2}=0.09, p=0.01\right)$, though not to body mass index (weight $(\mathrm{kg}) /$ height $\left(\mathrm{m}^{2}\right)$ ).

Figure 2 shows the distribution of sweatspot densities in normal and diabetic subjects. In contrast with the normal distribution found in normal subjects, there is an excess of diabetic subjects with low sweatspot counts, a decrease in the proportion with densities up to 1 SD above the normal range, and an excess in the range 104$132 / \mathrm{cm}^{2}$. Fourteen patients $(17.5 \%)$ had a mean density less than $20 / \mathrm{cm}^{2}$, and five $(6.3 \%)$ a density greater than $132 / \mathrm{cm}^{2}$, an overall rate of abnormality in this population of $23.8 \%$. The clinical characteristics of these groups are shown in Table 2 . Those with increased activation were significantly younger $(p=0.004)$ and had a longer duration of known diabetes; they were all Type 1 (insulin- dependent) diabetic patients. Only one had symptomatic neuropathy, but three had at least one abnormal (age-related) cardiovascular autonomic function test. Two had no neurophysiological abnormalities. Glycated haemoglobin was similar in the two groups, but those with high sweatspot counts had significantly higher median blood glucose levels at the time of the test. Eight of the 14 patients with low sweatspot densities had symptomatic autonomic neuropathy.

\section{Associations with other neurological tests}

Mean sweatspot density was weakly correlated with respiratory $\mathrm{RR}$ variation $\left(r^{2}=0.05, p=0.05\right.$, Fig. 3$)$ and Valsalva ratio $\left(r^{2}=0.09, p=0.03\right)$, but not with the $30: 15$ ratio $\left(r^{2}=0.007\right)$ nor with postural blood pressure change. There were no associations with mean sweatspot diameter. There was a striking lack of association between sweatspot measurements and thermal and heat pain thresholds (e.g. warm threshold and density: $r^{2}=-0.08, p=0.4$ ), but vibration perception thresholds were negatively correlated with mean sweatspot density (medial malleolus: $r^{2}=-0.11, p=0.001$, Fig. 4; great toe: $\left.r^{2}=-0.11, p=0.008\right)$. Motor nerve conduction measurements were not significantly associated with sweatspot measurements. The overall prevalence of abnormal sweatspot densities ( $24 \%$ ) was similar to that of common peroneal conduction velocity $(21 \%)$ and respiratory RR variation $(18 \%)$, and somewhat higher than that of the Valsalva ratio $(10 \%)$ and $30: 15$ ratio $(12 \%)$, though considerably lower than measurements of warm $(37 \%)$ and cool $(35 \%)$ thermal thresholds.

\section{Reproducibility}

To assess the variability of the computerised measurement method randomly-selected sweatspot prints of seven normal subjects and seven diabetic subjects were analysed on a different day. Median coefficient of variation (CV\%) (range) for sweatspot counts in normal sub-

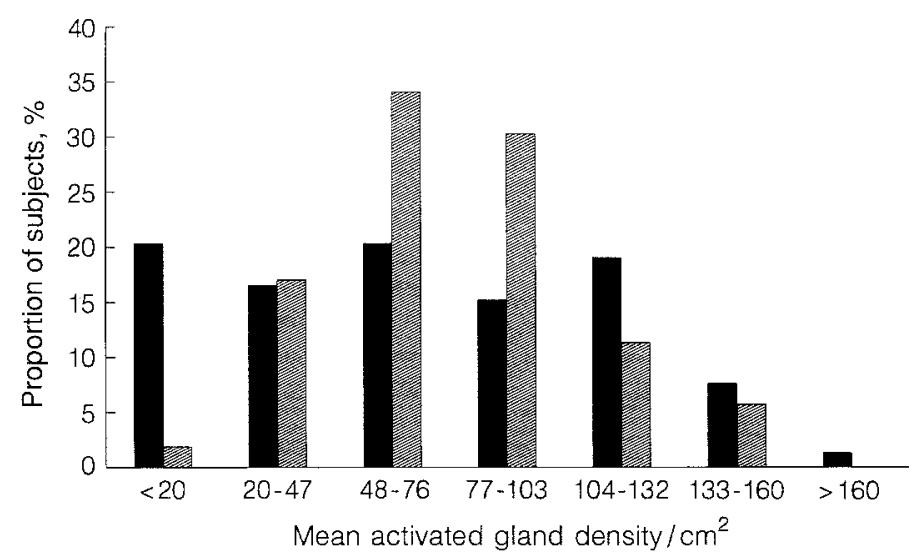

Fig. 2. Histogram of mean sweatspot density (bandwidth $1 \mathrm{SD}=27$ ) in diabetic and normal subjects. The relative excess of diabetic patients at the extremes of the distribution can be clearly seen.

diabetic patients; 霞 normal subjects 
Table 2. Comparison of patients with increased $(n=5)$ and decreased $(n=14)$ sweatspot activation

\begin{tabular}{|c|c|c|c|}
\hline & Increased & Decreased & $p$ \\
\hline$\overline{\text { Age (years) }}$ & $20(18-39)$ & $51(30-65)$ & 0.004 \\
\hline Diabetes duration (years) & $18(4-30)$ & $2.5(1-30)$ & 0.126 \\
\hline Type 1:Type 2 & $5: 0$ & $4: 10$ & \\
\hline $\begin{array}{l}\text { Respiratory RR variation, } \\
\text { (beats per min) }\end{array}$ & $23(13-29)$ & $11(3-32)$ & 0.047 \\
\hline Valsalva ratio & $1.91(1.84-2.24)$ & $1.09(0.98-1.44)$ & 0.011 \\
\hline $30: 15$ ratio & $1.11(0.96-1.37)$ & $1.09(0.98-1.44)$ & 0.734 \\
\hline Systolic BP fall $(\mathrm{mm} \mathrm{Hg})$ & $8(-10-12)$ & $7(-9-22)$ & 0.326 \\
\hline Diastolic BP fall (mm Hg) & $8(4-18)$ & $1(-15-22)$ & 0.176 \\
\hline $\begin{array}{l}\text { Marstock warm threshold } \\
\left({ }^{\circ} \mathrm{C}\right)\end{array}$ & $5.9(4.3-12.5)$ & $6.1(5.0-15.8)$ & 0.739 \\
\hline $\begin{array}{l}\text { Marstock cool threshold } \\
\left({ }^{\circ} \mathrm{C}\right)\end{array}$ & $4.1(1.7-12.0)$ & $6.0(1.4-7.2)$ & 1.000 \\
\hline $\begin{array}{l}\text { Random blood glucose } \\
(\mathrm{mmol} / \mathrm{l})\end{array}$ & $17.9(12.2-19.0)$ & $6.8(4.8-15.4)$ & 0.033 \\
\hline Glycated haemoglobin (\%) & $13.1(8.6-15.1)$ & $12.2(7.3-16.5)$ & 0.919 \\
\hline
\end{tabular}

$\mathrm{BP}$, blood pressure; Marstock threshold

jects was $14.1 \%(4.6-20.5 \%)$, and in diabetic subjects $9.5 \%(1.4-20.0 \%)$. Median CV for sweatspot size in normal subjects was $4.0 \%(0.6-6.0 \%)$, and in diabetic subjects $2.6 \%(0.7-4.0 \%)$.

Randomly-selected subjects (six normal and eight diabetic patients) had repeat tests on the same foot 2-24 weeks apart. Median CV for sweatspot density in normal subjects was $4.8 \%(2.3-25.4 \%)$, and in diabetic subjects $9.4 \%(4.3-49.4 \%)$. Coresponding results for sweatspot size were $5.5 \%(2.9-12.8 \%)$ in normal subjects and $9.1 \%(0.5-20.3 \%)$ in diabetic subjects.

\section{Discussion}

This study has simplified and automated the process of counting and measuring sweatspots in normal and diabetic subjects, and has shown that although some of the correlations between somatic and autonomic nerve function and pilocarpine-activated sweatspot densities are statistically significant, they are generally very weak. Kennedy and Navarro [12] recently reported their experience using an image analysis similar to that described here, and found that the increased precision resulted in a greater diagnostic yield. However, neither this study, nor that of Ryder et al. [10], using injected acetylcholine, identified patients with increased sweatspot activation.

The reasons for increased sweatspot activation are not known. It occurs in a few patients with Type 1 diabetes, some of whom have evidence of abnormal nerve function, but not to the extent of the larger group with decreased sweatspot activation. In a sample of 79 diabetic patients a small number of non-neuropathic patients would be expected to have sweatspot densities above the $95 \%$ confidence interval, and there were two subjects who had no abnormalities in the other neurological tests. In the remainder of the subjects, we assume that the phenomenon is due to cholinergic denervation supersensitivity, but the mechanisms involved are not known, nor are the reasons why similar patients do not exhibit the same phenomenon.

The actual density of sweat glands in this region of the dorsum of the foot is not known. However, comparative histological and functional studies have been conducted on the skin of the thigh, where histological densities of up to $120 / \mathrm{cm}^{2}$ have been found [17]; methacholine-activated sweatspot counts are much lower, ranging from $45-86 / \mathrm{cm}^{2}$ [18], though a larger number is activated by heat stress [19]. Therefore, the number of potentially activatable glands is much greater than the number stimulated by pharmacological or physiological stimuli. The use of higher currents (or increased pilocarpine concentrations) in our study may have resulted in higher sweatspot den-

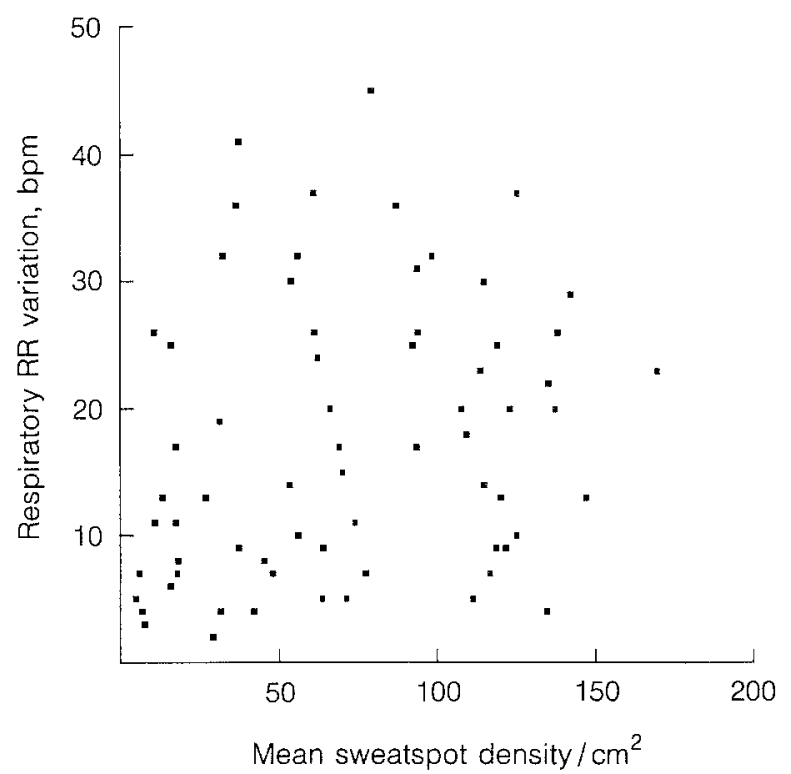

Fig. 3. Scatterplot showing relation between respiratory $R R$ variation and sweatspot density in diabetic subjects $\left(r^{2}=0.05, p=0.05\right)$

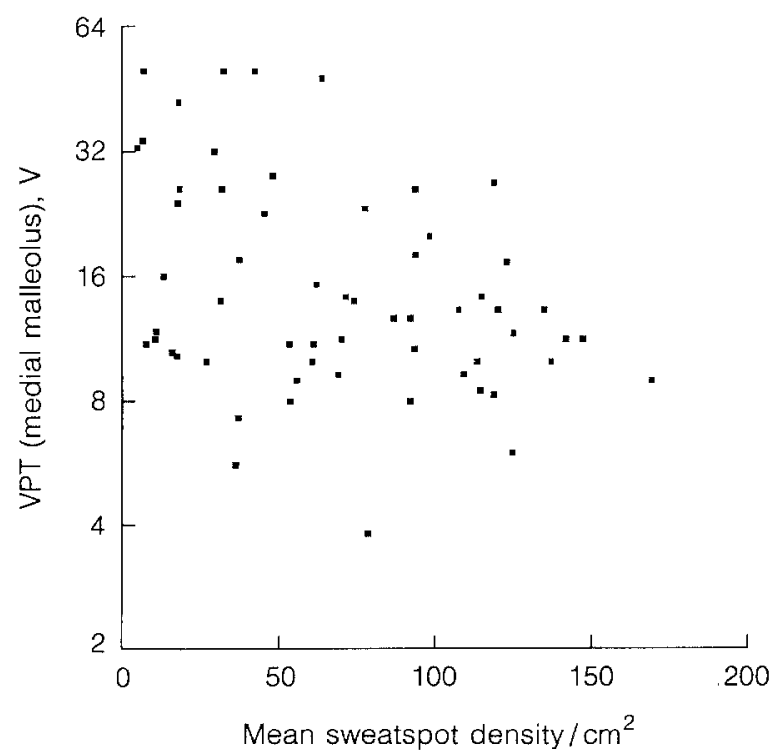

Fig.4. Scatterplot showing relation between vibration perception threshold (VPT) at the medial malleolus and sweatspot density in diabetic subjects $\left(r^{2}=0.11, p=0.004\right)$ 
sities, but we employed a clinically safe level that is in widespread use and that results in optimum sweat output in cystic fibrosis testing [20]. In the presence of denervation supersensitivity otherwise inactive glands with a high stimulation threshold would be recruited by a given cholinergic stimulus. The standardisation of the quantity of applied pilocarpine and current, together with the stimulation of a large area of the foot $\left(4 \mathrm{~cm}^{2}\right)$, and the use of triplicated measurements over an identical area on three occasions at 12,16 and 20 min, may explain why increased sweatspot densities have been detected in this study. Until repeated measurements over time have been made it is not possible to say for how long this presumed supersensitive state persists, nor whether it precedes or follows subnormal sweat gland activation. Since pilocarpine is a muscarinic agent acting directly on sweat gland epithelium, and not through neuronal pathways [21], it is probable that increased responses reflect upgrading of cholinergic sweat gland receptors; histological studies will be required to determine whether these changes are associated with changes in sweat gland innervation. There is preliminary evidence, however, that some diabetic patients have increased sweat-gland immunoreactive nerve areas in skin biopsies [22]. Low et al. [11,23] also observed increased sweat output after iontophoresis with acetylcholine, in a system which stimulated only nicotinic cholinergic receptors, and therefore changes in nerves as well as in the target organ would be expected. The group of supersensitive patients in the current study is heterogeneous, unlike the clearly neuropathic patients with decreased sweatspot activation. In some there may be physiological reasons for these increased responses, for example sweat gland training through environmental exposure [24] or physical training [25]; the explanation that iontophoresis is more effective in the skin of young people is unlikely in view of our finding that there was no change in sweatspot density with age in our normal group.

Increased sweatspot activation was associated with raised random blood glucose taken at the end of the test. No patients were hypoglycaemic before the test, and therefore "rebound" hyperglycaemia after hypoglycaemia, causing increased sweat gland activation, cannot be the explanation for this phenomenon. Hyperglycaemia is not associated with increased activity in skin sudomotor fibres [26], and in the diabetic patients there were no significant correlations between sweatspot measurements and either blood glucose or glycated haemoglobin. Elevated blood glucose in this small group of patients may therefore be a chance finding.

Sweatspot density correlated negatively with several neurophysiological tests, but the statistical weakness of these associations compared with the strong associations generally found between neurophysiological measures $[27,28]$ suggests that peripheral denervation of sweat glands differs from the deficits in the long neural pathways generally tested by conventional neurophysiological methods. The current test, therefore, cannot be used as a substitute for standard tests, and should be regarded as complementary to standard neurophysiology by reflecting events at the most distal autonomic nerve terminals. This view is strengthened by the lack of correlation between thermal thresholds and sweatspot activation, though both are mediated through small-fibres. Navarro et al. [11], using a categorical clinical score of temperature sensitivity, found a good association between thermal deficit and decreased sweatspot activation. The stronger negative correlation between vibration perception thresholds and sweatspot density cannot readily be explained, particularly in view of the evidence that vibration perception is transmitted through myelinated fibre pathways.

The method described here has acceptable reproducibility, similar to that of pupillometry [29] and vagal cardiovascular autonomic tests [30]. In this randomly-selected population, sweatspot activation was at least as frequently abnormal as other commonly used neurological tests, apart from thermal threshold measurements, and therefore has a similar diagnostic sensitivity. There is no evidence from this study that local sweatspot activation is more frequently abnormal than other tests, and does not confirm the widely-held clinical viewpoint that peripheral small-fibre function is preferentially affected in diabetic neuropathy $[31,32]$. This technique is the simplest method currently available for evaluating distal sudomotor function, as it is rapid, simple, and objective, and offers a method for identifying a group of diabetic patients with increased sweat gland activation, together with a group of unequivocally neuropathic patients with decreased activation. We suggest that the test may be of value in monitoring the response of therapeutic agents, such as ACTH fragments and gangliosides, which experimentally have been found to increase peripheral nerve sprouting, and whose effects may not be adequately reflected in conventional neurophysiological measurements.

Acknowledgements. We thank Dr. S.McHardy-Young for having allowed us to study his patients. We are grateful to T. Kelly, H.Holland and Ms. P.del Grazia of Cambridge Instruments, Cambridge UK, for help with programming the Quantimet 970 image-analyser and for allowing access to their equipment.

\section{References}

1. Kennedy WR, Sakuta M (1984) Collateral reinnervation of sweat glands Ann Neurol 15:73-78

2. Kennedy WR, Navarro X, Kamei H (1988) Reinnervation of sweat glands in the mouse: axonal regeneration versus collateral sprouting. Muscle Nerve 11: 603-609

3. List CF, Peet MM (1930) Sweat secretion in man. III. Clinical observations on sweating produced by pilocarpine and mecholyl (acetyl-beta-methylcholine chloride) in man. Arch Neurol Psychiat 40: 269-290

4. Simeone FA, Mentha C, Rodrigues HA (1951) Responsiveness of sweat glands after denervation by preganglionic ramisection, ganglionectomy and peripheral nerve section. Am J Physiol 165: 356-364

5. Montagna W (1962) Histological, histochemical and pharmacologic properties. In: Montagna W, Ellis RA, Silver A (eds) Advances in biology of skin. Vol III: Eccrine sweat glands and eccrine sweating. Pergamon Press Oxford pp 6-29

6. Silver A, Versuci W, Montagna W (1964) Studies of sweating and sensory function in cases of peripheral nerve injuries to the hand. J Invest Dermatol 43: 255-266

7. Kennedy WR, Sakuta M, Sutherland D, Goetz FC (1984) The sweating deficiency in diabetes mellitus: methods of quantitation and clinical correlation. Neurology (Cleveland) 34: 758-763 
8. Ryder REJ, Marshall R, Johnson K, Ryder AP, Owens DR, Hayes TM (1988) Acetylcholine sweatspot test for autonomic denervation. Lancet I: 1303-1305

9. Navarro X, Kennedy WR, Fries TJ (1989) Small nerve fiber dysfunction in diabetic neuropathy. Muscle Nerve 12: 498-507

10. Kennedy WR, Navarro X (1989) Sympathetic sudomotor function in diabetic neuropathy. Arch Neurol 46: 1182-1186

11. Low PA, Caskey PE, Tuck RR, Fealey RD, Dyck PJ (1983) Quantitative sudomotor axon reflex test in normal and neuropathic subjects. Ann Neurol 14: $573-580$

12. Herrmann F, Prose PH, Sulzberger MB (1951) Studies on sweating. IV. A new quantitative method of assaying sweat-delivery to circumscribed areas of the skin surface. J Invest Dermatol 17: 241-249

13. Abraham RR, Abraham RM, Wynn V (1986) Autonomic and electrophysiological studies in patients with signs or symptoms of diabetic neuropathy. Electroencephalogr Clin Neurophysiol 63: 223-230

14. Bloom S, Till S, Sönksen P, Smith S (1984) Use of a biothesiometer to measure individual vibration thresholds and their variation in 519 non-diabetic subjects. Br Med J 288: 1793-1795

15. Levy D, Abraham R, Reid G (1989) A comparison of two methods for measuring thermal thresholds in diabetic neuropathy. J Neurol Neurosurg Psych 52: 1072-1077

16. Ewing DJ, Clarke BF (1982) Diagnosis and management of diabetic autonomic neuropathy. Br Med J 285: 916-918

17. Szabo $G$ (1962) The number of eccrine sweat glands in human skin. In: Montagna W, Ellis RA, Silver AF, (eds) Advances in biology of skin, Vol III: Eccrine sweat glands and eccrine sweating. Pergamon Press Oxford pp. 1-5

18. Kenney WL, Fowler SR (1988) Methylcholine-activated eccrine sweat gland density and output as a function of age. J Appl Physiol 65: 1082-1086

19. Dobson RL, Sato K (1972) The stimulation of eccrine sweating by pharmacological agents. In: Montagna W, Van Scott EJ, Stoughton RB (eds) Advances in the biology of skin, Vol XII. Pharmacology and the skin. Appleton-Century-Crofts New York, pp 447-475

20. Webster HL (1983) Laboratory diagnosis of cystic fibrosis. CRC Crit Rev Clin Lab Sciences 18:313-338

21. Randall WC, Kimura KK (1955) The pharmacology of sweating. Pharmacol Rev 7: 365-397

22. Levy DM, Springall D, Terenghi G, Reynolds G, Abraham RR, Polak JM (1990) Differential changes in a general neuronal mar- ker and neuropeptides in diabetic skin. Diabetologia 33: A16 (Abstract)

23. Low PA, Zimmerman BR, Dyck PJ (1986) Comparison of distal sympathetic with vagal function in diabetic neuropathy. Muscle Nerve 9: 592-596

24. Collins KJ, Crockford GW, Weiner JS (1965) Sweat-gland training by drugs and thermal stress. Arch Environ Health 11: 407422

25. Sato K, Sato F (1983) Individual variations in structure and function of human eccrine sweat gland. Am J Physiol (RICP 14) 245 : R203-R208

26. Berne C, Fagius J, Niklasson F (1989) Sympathetic response to oral carbohydrate administration. Evidence from microelectrode nerve recordings. J Clin Invest 84: 1403-1409

27. Pfeifer MA, Weinberg CR, Cook DL et al. (1985) Correlations among autonomic, sensory, and motor neural function tests in untreated non-insulin-dependent diabetic individuals. Diab Care 8: 576-584

28. Abraham RR, Levy DM (1990) Diabetic neuropathy: measurement and quantitation. In: Ward J, Goto Y (eds) Diabetic neuropathy. Wiley \& Sons, Chichester, pp 259-268

29. Smith SA, Dewhirst RR (1986) A simple diagnostic test for pupillary abnormality in diabetic autonomic neuropathy. Diab Med 3: 38-41

30. Schumer M, Miller-Crain G, Pfeifer MA, The Statil Study Group (1988) Diabetic autonomic neuropathy - Part II. Coefficient of variation of RR-variation and Valsalva maneuver tests. Am J Med 85 [Suppl 5A]: 144-146

31. Lawrence RD (1965) The diabetic life: a concise practical manual, 12th edn. Churchill, London p 157

32. Ewing DJ, Clarke BF (1986) Diabetic autonomic neuropathy: present insights and future prospects. Diab Care 9: 648-665

Received: 13 May 1991

and in revised form: 5 August 1991

Dr. D. Levy

Department of Diabetes and Endocrinology

Central Middlesex Hospital

Acton Lane

Park Royal

London NW10 7NS

UK 